\title{
Frequency and significance of errors related to parenteral nutrition in children
}

\author{
P. Narula ${ }^{1}$, D. Hartigan ${ }^{2}$ and J. W. L. Puntis ${ }^{1}$ \\ ${ }^{1}$ Department of Paediatric Gastroenterology and Nutrition and ${ }^{2}$ Paediatric Nutrition Pharmacist, Leeds General Infirmary, \\ Leeds LS13BR, UK
}

Providing parenteral nutrition (PN) is a complex process with the potential for harmful errors. There is little information available on the frequency and significance of such errors.

The aim of this study was to determine the nature, frequency and significance of errors related to the PN process in a tertiary paediatric unit.

In our children's centre, it is policy that 'any unexpected event with an actual or potential detrimental effect on a patient is formally reported on an incident (IR1) form' by staff. We therefore reviewed all IR1 forms related to PN between January06 and June09. The errors were categorised according to where in the PN process they occurred. Harm scores (severity of the error in relation to patient safety) were based on the framework of the National Coordinating Council for Medication Error Reporting and Prevention (NCC MERP).

Over $18588 \mathrm{PN}$ days, 46 errors were identified, giving an error frequency of $0.24 \%$. Of these, $5(11 \%)$ occurred during the prescription process (e.g. incorrect PN volume calculation), 9(20\%) during the transcription process (e.g. incorrect patient ID, surname, date of birth, rates or inadequate amount of $\mathrm{PN}), 11(24 \%)$ during dispensing (e.g. mislabelling of vamin or lipid bags, leaking bags), 7 (15\%) during delivery of PN to the ward and $14(30 \%)$ during the administration process (e.g. PN administered at incorrect rates). No errors were reported during the preparation/compounding process. Forty-three $(94 \%)$ errors did not result in patient harm, while $3(6 \%)$ errors resulted in temporary harm (1 PN extravasation; 1 incorrect infusion rate in a newborn resulting in hyperglycaemia and fluid overload, 1 incorrectly high potassium dosage in PN).

PN-related errors resulting in harm appear to be rare. Most occur during dispensing and administration suggesting that more robust checking procedures may be required during these phases. The widespread reporting of non-harmful errors indicate that staff have an appropriately low threshold for completing IR1 forms, and that these represent a valuable audit tool for improving patient safety. 